\begin{tabular}{c|c|c}
\hline \hline Vol. 269: 153-162, 2004 & MARINE ECOLOGY PROGRESS SERIES \\
Mar Ecol Prog Ser & Published March 25 \\
\hline
\end{tabular}

\title{
Zooplankton use of chemodetection to find and eat particles
}

\author{
George A. Jackson ${ }^{1, *}$, Thomas Kiørboe ${ }^{2}$ \\ ${ }^{1}$ Department of Oceanography, Texas A\&M University, College Station, Texas 77843-3146, USA \\ ${ }^{2}$ Danish Institute for Fisheries Research, Kavalergården 6, 2920 Charlottenlund, Denmark
}

\begin{abstract}
The ability of raptorial zooplankton to find large particles such as marine aggregates is crucial to their use of the particles as food and to the fate of the particles. Kiørboe \& Thygesen (2001) developed a numerical approach to describe particle detection by chemosensory zooplankton. In this paper, we develop and test a simplified mathematical description of the process and explore the ecological implications of chemosensory particle detection. Our results suggest that chemosensory particle detection can be more efficient than hydrodynamic detection. The exact extent depends greatly on the sensitivity of chemodetection in zooplankton, a process that has not been well studied experimentally.
\end{abstract}

KEY WORDS: Marine snow $\cdot$ Zooplankton feeding $\cdot$ Plumes $\cdot$ Hydromechanical signals $\cdot$ Chemical signals Resale or republication not permitted without written consent of the publisher

\section{INTRODUCTION}

Once believed to consist of simple sieving of water to remove food particles, zooplankton feeding is now known to involve sophisticated sensing of the environment as the organism looks for environmental perturbations induced by the food. The traces left by a falling particle range from disruptions of the fluid flow to chemical trails. The ability of an organism to sense its environment determines its ability to eat and to avoid being eaten, and determines where it lives.

Understanding the detection of environmental cues requires a description of the physical and chemical effects of a particle (including the organism itself) and its movement as well as observations of organism sensory responses. The nature of fluid-mechanical interactions have been best described for moving animals (e.g. Kiørboe \& Visser 1999, Titelman 2001) and for animals in turbulent environments. Chemical cues have been better studied for microorganisms looking for favorable environments, including falling aggregates (Jackson 1989, Blackburn et al. 1998).

Thygesen \& Kiørboe (2002) developed a numerical technique to calculate the hydrodynamic and chemical regime around a falling aggregate with a Reynolds number $R e<20$, that is leaking organic material as it falls. Kiørboe \& Thygesen (2001) applied the technique to show that the chemical plume around the particle could enhance the ability of animals to find and consume the aggregate, but they did not systematically explore its implications. The high computational cost makes such an exploration difficult.

Here we develop a simplified analytical model of the formation of the plume behind a moving and leaking particle, and we use the result to calculate the ability of a chemosensory animal to find the particle. Note that such search strategies do not apply to all zooplankton, as pure filter feeders such as salps and flux feeders such as pteropods are not known to actively search for particles. We compare our analytical results to those from the more rigorous numerical simulations and use these to render the analytical result more accurate. We then compare the efficiency of animals with different search behaviors in finding falling particles by different sensory mechanisms.

Because the ability of zooplankton to find and consume falling aggregates depends on the sizes of the animals as well as on the particles, we initially focus on 
describing the factors associated with the detection of a single particle by a single animal. We then extend the analysis to include the distribution of particles over a range of sizes. A list of the symbols used is given in Table 1.

\section{CROSS-SECTION OF THE PLUME}

\section{Perspective of a point-sized copepod}

We start by calculating the cross-sectional area that a particle falling vertically presents to an animal swimming horizontally. While the particle has a finite size and spreads out water passing near it, this water comes together again on the upstream side. We will assume that the particle acts as a point source (infinitesimally small spatial extension). Because the largest concentration gradients are perpendicular to the direction of movement, diffusion in the direction of motion is less important than in the perpendicular directions. For simplicity, we will ignore the former unless otherwise noted. We will further assume that

Table 1. Notation

\begin{tabular}{|c|c|c|}
\hline Symbol & Description & Dimensions \\
\hline$a, b$ & Constants for $n(r)$ & $\mathrm{cm}^{-4+b}$ \\
\hline$c, d$ & Constants for $v(r)$ & $\mathrm{cm}^{1-d} \mathrm{~s}^{-1}$ \\
\hline$C(z)$ & Concentration at boundary of plume & $\mathrm{mol} \mathrm{cm}^{-3}$ \\
\hline$C_{0}$ & $\begin{array}{l}\text { Threshold detection concentration for } \\
\text { plume }\end{array}$ & $\mathrm{mol} \mathrm{cm}^{-3}$ \\
\hline$D$ & Diffusion coefficient & $\mathrm{cm}^{2} \mathrm{~s}^{-1}$ \\
\hline$e, f$ & Constants for $L(r)$ & $\mathrm{mol} \mathrm{cm}^{-f} \mathrm{~s}^{-1}$ \\
\hline$F(r)$ & Food consumption rate by zooplankter & $\mathrm{mol} \mathrm{s}^{-1}$ \\
\hline$g(r)$ & Food consumption per contact & mol \\
\hline$g_{0}$ & Fixed food consumption per contact & $\mathrm{mol}$ \\
\hline 1 & Specific leakage rate & $\mathrm{s}^{-1}$ \\
\hline$L(r)$ & Leakage rate & $\mathrm{mol} \mathrm{s}^{-1}$ \\
\hline$m(r)$ & Particle mass & mol \\
\hline$n(r)$ & Particle size spectrum & $\mathrm{cm}^{-4}$ \\
\hline$r$ & Particle radius & $\mathrm{cm}$ \\
\hline$r_{Z}$ & Zooplankter radius & $\mathrm{cm}$ \\
\hline$S$ & $\begin{array}{l}\text { Sensory distance from center of } \\
\text { zooplankter }\end{array}$ & $\mathrm{cm}$ \\
\hline$u_{*}$ & Threshold signal strength & $\mathrm{cm} \mathrm{s}^{-1}$ \\
\hline$V(r)$ & Aggregate fall velocity & $\mathrm{cm} \mathrm{s}^{-1}$ \\
\hline$v_{Z}$ & Zooplankton swimming speed & $\mathrm{cm} \mathrm{s}^{-1}$ \\
\hline$v_{Z H}$ & Horizontal component of $v_{Z}$ & $\mathrm{~cm} \mathrm{~s}^{-1}$ \\
\hline$z$ & Vertical distance from aggregate & $\mathrm{cm}$ \\
\hline$Z$ & Length of plume, where $C(Z, 0)=C_{0}$ & $\mathrm{~cm}$ \\
\hline$\beta$ & Encounter rate kernel & $\mathrm{cm}^{3} \mathrm{~s}^{-1}$ \\
\hline$\rho(z)$ & Plume radius & $\mathrm{cm}$ \\
\hline$\sigma_{0}$ & Plume cross-section & $\mathrm{cm}^{2}$ \\
\hline$\sigma_{0, \text { fit }}$ & Plume cross-section fit to $\sigma_{0, \text { num }}$ & $\mathrm{cm}^{2}$ \\
\hline$\sigma_{0, \text { num }}$ & $\begin{array}{l}\text { Plume cross-section calculated } \\
\text { numerically }\end{array}$ & $\mathrm{cm}^{2}$ \\
\hline$\sigma_{0, \mathrm{pt}}$ & $\begin{array}{l}\text { Plume cross-section calculated from } \\
\text { analytical approximation }\end{array}$ & $\mathrm{cm}^{2}$ \\
\hline$\sigma_{\mathrm{s}}$ & Additional sensory cross-section & $\mathrm{cm}^{2}$ \\
\hline$\sigma$ & Total cross-section & $\mathrm{cm}^{2}$ \\
\hline
\end{tabular}

the velocity field around the falling particle has no effect in moving the material once it is released, so that the description of the solute distribution is a pure diffusion problem.

The concentration enhancement $C$ associated with leakage from the particle is then described by:

$$
v \frac{\partial C}{\partial z}=D\left(\frac{\partial^{2} C}{\partial x^{2}}+\frac{\partial^{2} C}{\partial y^{2}}\right)
$$

where the fall velocity $v$ is in the vertical $(z)$ direction and $D$ is the diffusion coefficient. If the aggregate releases material at a rate $L$, then the release rate per unit distance is related to the sinking velocity: $L / V$. (Notice that the coordinate system has been transformed so that the particle is fixed and $z$ is actually the distance to the particle.) The resulting equation has the solution:

$$
C=\frac{L}{4 \pi D z} \exp \left(-\frac{v\left(x^{2}+y^{2}\right)}{4 D z}\right)=\frac{L}{4 \pi D z} \exp \left(-\frac{v \rho^{2}}{4 D z}\right)
$$

where $C$ is the concentration enhancement above any background concentration and $\rho$ is the distance away from the plume centerline (e.g. Okubo 1980, but modified by the absence of a reflecting boundary: Fischer et al. 1979).

The enhancement at which the concentration is too small for an animal to detect, $C_{0}$, determines the bounds of the plume. We can estimate the effective length of the plume $Z$, at which $C=C_{0}$ on the $z$-axis $(\rho=0$; Fig. 1$)$, as:

$$
Z=\frac{L}{4 \pi D C_{0}}
$$

The plume radius $\rho^{*}$ at any distance $z$ can be calculated as the horizontal distance from the particle path to the location at which $C=C_{0}$ using Eq. (2):

$$
\begin{aligned}
\rho^{*} & =\left(\frac{4 D z}{V}\right)^{0.5}\left(\ln \frac{L}{4 \pi D C_{0} Z}\right)^{0.5} \\
& =\left(\frac{4 D z}{V}\right)^{0.5}(-\ln z / Z)^{0.5}
\end{aligned}
$$

The cross-sectional area of the plume is $\sigma_{0}$; the value estimated with this point source approximation, $\sigma_{0, \mathrm{pt}}$, is then given by:

$$
\begin{aligned}
\sigma_{0, \mathrm{pt}} & =2 \int_{0}^{Z}\left(\frac{4 D z}{V}\right)^{0.5}(-\ln Z / z)^{0.5} \mathrm{~d} z \\
& =2\left(\frac{4 D Z}{V}\right)^{0.5} Z \int_{0}^{1} z^{\prime 0.5}\left(-\ln z^{\prime}\right)^{0.5} \mathrm{~d} z^{\prime} \\
& =\frac{0.24}{D v^{0.5}}\left(\frac{L}{\pi C_{0}}\right)^{1.5}
\end{aligned}
$$

where $z=z^{\prime} Z$ and $\int_{0}^{1} z^{\prime 0.5}\left(-\ln z^{\prime}\right)^{0.5} \mathrm{~d} z^{\prime}=\sqrt{\frac{2 \pi}{27}}=0.481$. 


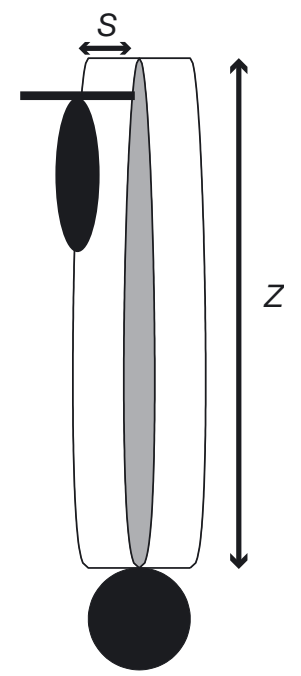

Fig. 1. Diagram of plume behind a falling particle. The effective cross-section of the plume can be increased by the width of the sensory apparatus

\section{Perspective of a finite size animal}

If the animal has its antennae out a distance $S$ from its center and can sense anything within $S$, the effect is to increase the width of the plume by $S$ on either side, for a total of $2 S$. Hence, the total detection crosssection for an animal becomes:

$$
\begin{aligned}
\sigma & =\int_{0}^{Z} 2\left(\rho^{*}+S\right) \mathrm{d} z \\
& =\sigma_{0}+2 S Z \\
& =\sigma_{0}+2 S \frac{L}{\pi D C_{0}} \\
& =\sigma_{0}+\sigma_{s}
\end{aligned}
$$

length of the detectable plume for a $0.25 \mathrm{~cm}$ radius particle is as long as $99 \mathrm{~cm}$ and the cross-section for the point animal is $20 \mathrm{~cm}^{2}$. This value of $\sigma_{0, p t}$ is considerably larger than that of the particle by itself, here $0.19 \mathrm{~cm}^{2}$. The plume length is well predicted by Eq. (3) over the whole particle size range (Fig. 2A) but the value of $\sigma_{0}$ underestimates the results from the numerical simulations for large $r$ (Fig. 2B).

A more detailed comparison requires the normalization of the above equations (length using $r$, time using $r / v$ and mass using $L r / v, C^{\prime}=C_{0} v r^{2} / L, P e=v r / D=$ Peclet number). The resulting equations are:

$$
\begin{aligned}
& Z_{\mathrm{pt}}^{\prime}=\operatorname{Pe}\left(4 \pi C^{\prime}\right)^{-1} \\
& \sigma_{0, \mathrm{pt}}^{\prime}=0.24 \operatorname{Pe}\left(\pi C^{\prime}\right)^{-1.5}
\end{aligned}
$$

where the subscript indicates that these are for the point model and the primes that these are normalized.

The simplified model again works well to predict the normalized plume length (Fig. 3A). The predictions of $\sigma_{0}$ for the simplified equations become inaccurate for $P e>10^{3}$, equivalent to $R e>1$ (Fig. 3B). Particles with $R e>1$ include large marine snow particles. The point approximation also diverges from the numerical solution for high values of $C^{\prime}$, equivalent to large leakage rates. That is, the values of $\sigma_{0, p t}$ are too small for high $R e$ and too large for high $C^{\prime}$.

We adjusted the analytical results for $\sigma_{0}^{\prime}$ by fitting a curve to the anomalies for high values of $\operatorname{Re}$ (Fig. 4):

$$
\begin{array}{rr}
\sigma_{0, \mathrm{fit}}^{\prime}=0.24 P e\left(\pi C^{\prime}\right)^{-1.5}\left(1+1.98 \times 10^{-7} P e^{1.97} C^{\prime-1.33}\right) \\
\text { for } 10>R e \geq 1 \\
=0.24 P e\left(\pi C^{\prime}\right)^{-1.5} & \text { for } R e<1
\end{array}
$$

where $\sigma_{s}=2 S Z$ is the extra cross-sectional area associated with the animal's size.

\section{Comparison with numerical solutions}

The above solutions are for a point particle that does not disturb water flow; real aggregates are not points and they do perturb the water flow. The added complexity of flow around particles has been addressed using numerical solutions of the relevant advective-diffusive equations. We make a more systematic comparison with the results of such numerical calculations using the program of Thygesen \& Kiørboe (2002).

Results are shown for particles with the properties of typical marine snow particles (e.g. Kiørboe \& Jackson 2001) and chemosensory capability of zooplankters (here with $C_{0}=10^{-9} \mathrm{~mol} \mathrm{~cm}^{-3}$ ) (Fig. 2). For this set of properties, the
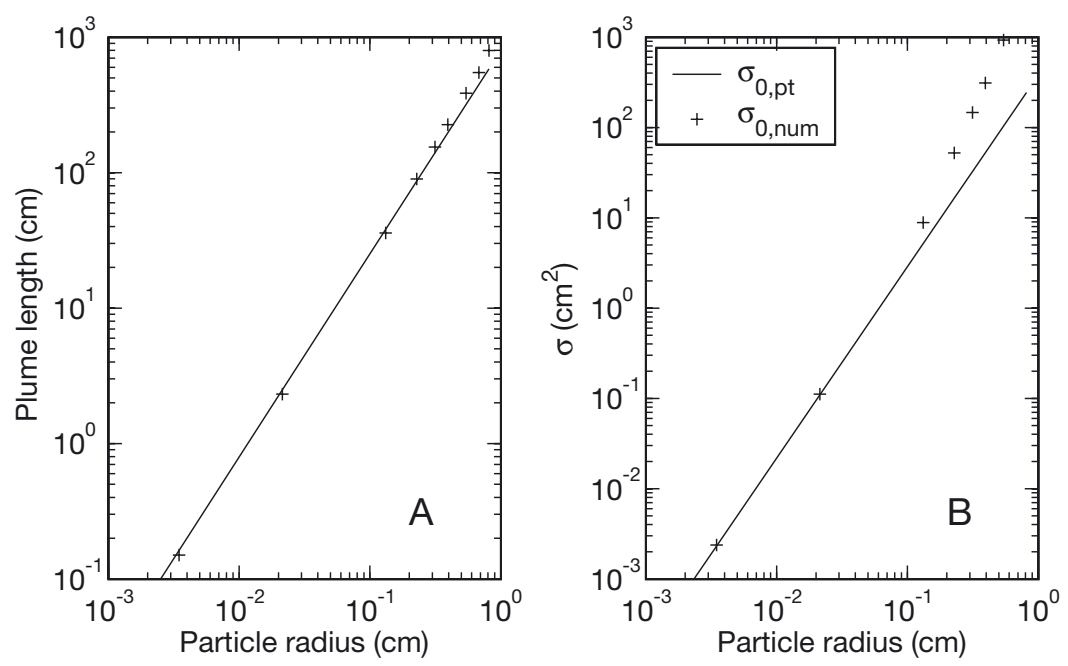

Fig. 2. Effect of particle size on length of plume $Z$ and plume cross-section $\sigma: V=0.13 r_{p}^{0.26}, L=1 \times 10^{-12} r_{p}^{1.5}$, and $C_{0}=1 \times 10^{-9} \mathrm{~mol} \mathrm{~cm}^{-3}$. (A) plume length calculated from numerical simulations (+), and Eq. 3 (solid line); (B) plume cross sections: $\sigma_{0, p t}$ (solid line), and $\sigma_{0, \text { num }}$ calculated using the method of Kiørboe \& Thygesen $(2001 ;+)$ 
In dimensional form, these become:

$$
\begin{aligned}
& \sigma_{0, \mathrm{fit}}=\frac{0.24}{D v^{0.5}}\left(\frac{L}{\pi C_{0}}\right)^{1.5}\left(1+1.98 \times 10^{-7} \frac{V^{0.64} L^{1.33}}{r^{0.69} C_{0}^{1.33} D^{1.97}}\right) \\
&=\frac{0.24}{D v^{0.5}}\left(\frac{L}{\pi C_{0}}\right)^{1.5} \quad \text { for } 10>R e \geq 1 \\
& \text { for } R e<1
\end{aligned}
$$

Eq. (10) was used in subsequent calculations.

\section{Comparison with other feeding mechanisms}

In addition to plume-finding, animals have other ways of sensing falling particles. Visser (2001) has ana-
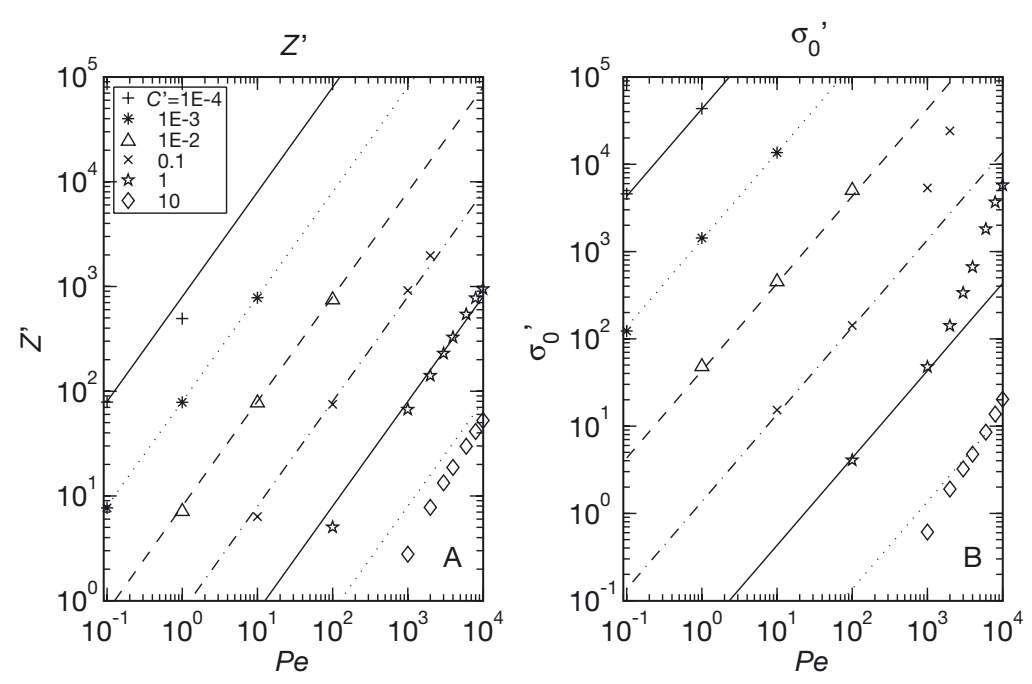

Fig. 3. Normalized and numerical values of (A) $Z^{\prime}$ and (B) $\sigma_{0}{ }^{\prime}$ as a function of $P e$. Lines represent the values of $Z_{\mathrm{pt}}^{\prime}$ and $\sigma_{0, \mathrm{pt}}^{\prime}$ for a given value of $C^{\prime}$ and symbols represent the results from numerical computations. Differences arise for $P e>10^{3}$, equivalent to the Reynolds number $R e>1$, and for high values of $C^{\prime}$

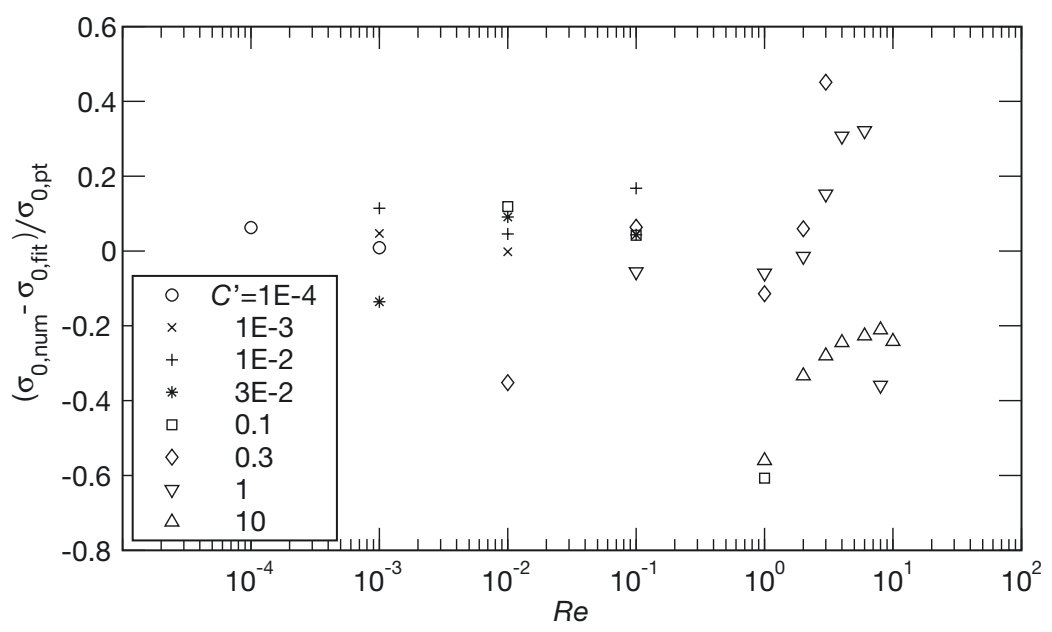

Fig. 4. Errors after fitting procedure. For the 17 data points with $R e \geq 1$, the mean error for the uncorrected fit was $\left(\frac{1}{17} \sum\left(\sigma_{0, \text { num }}^{\prime}-\sigma_{0, \mathrm{pt}}^{\prime}\right)^{2} / \sigma^{\prime 2}{ }_{0, \mathrm{pt}}\right)^{0.5}=14.8$; the mean error calculated using Eq. (9) was $\left(\frac{1}{17} \sum\left(\sigma_{0, \text { num }}^{\prime}-\sigma_{0, \mathrm{fit}}^{\prime}\right)^{2} / \sigma^{\prime 2}{ }_{0, \mathrm{pt}}\right)^{0.5}=$ 1.29. The legend provides the values of $C^{\prime}$ represented by each plotting symbol. The fitting procedure reduced the error for the points with $R e \geq 1$ to values similar to those for points with $R e<1$ lyzed the sensory efficiency for ambush feeding (in which a motionless animal waits for a hydromechancue) and cruising (in which an animal searches hydrodynar passage. The efficiencies of the different particle encounter mechanisms are best compared by considering the respective encounter rate kernels. For plume-finding, this is:

$$
\beta_{\text {plume }}=v_{Z H}\left(\sigma_{0}+\sigma_{s}\right)
$$

where $v_{Z H}$ is the horizontal component of the swimming velocity $\left(=0.82 v_{Z}\right.$, assuming isotropic swimming speeds and $v_{Z}$ is the zooplankton swimming speed). For the ambush feeder, it is:

$$
\beta_{\text {ambush }}=\pi S_{\mathrm{am} V}^{2}
$$

where $S_{\mathrm{am}}$ is the sum of the physical radius of the particle and sensory radius of the animal. For the cruising animal, the encounter rate kernel is:

$$
\beta_{\text {cruise }}=\pi S_{\text {cr }}^{2}\left(v_{Z}^{2}+v^{2}\right)^{0.5}
$$

where $S_{\text {cr }}$ is the appropriate sensory radius, the distance from the center of mass of the animal to the center of the particle. Since both cruisers and ambushers are utilizing hydromechanical signals, assume for convenience that they are equal: $S_{\mathrm{am}}=S_{\mathrm{cr}}$.

Flux feeding, in which the animal passively collects the particles that fall on a feeding structure, is an additional particle feeding mechanism (Jackson 1993). For the flux feeder:

$$
\beta_{\text {flux }}=\pi S_{\text {flux }}^{2} V
$$

Because the form of $\beta_{\text {flux }}$ is identical to that of the ambush feeder, we will not consider it further here.

If the particle is very small relative to the animal, then it is the fluid velocity generated by the particle which provides the relevant cue; if the particle is much larger than the animal, it is the fluid deformation that matters (Kiørboe \& Visser 1999). Visser (2001) developed an expression that considers both of these extremes as well as the transition:

$$
S_{\mathrm{cr}}=r_{Z}+0.5 r_{Z}\left[\left(1+3 \frac{r v}{r_{Z} u_{*}}\right)^{0.5}-1\right]
$$

where $r_{Z}$ is the animal radius and $u_{*}$ is the threshold signal strength required to elicit an attack response (estimated as $100 \mu \mathrm{m} \mathrm{s}^{-1}$; Titleman 2001, Lenz \& Hartline 
1999). (Note that this predicts a larger sensory distance than the simpler but less accurate formula used by Kiørboe \& Thygesen 2001). We have assumed that the length of a sensory appendage away from the center of the animal is the same as the animal radius $r_{Z}$.

Note that $\beta_{\text {cruise }}>\beta_{\text {ambush }}$ because the swimming provides an additional velocity component. In order to focus on the differences between the hydrodynamic and chemical sensing rates, we will focus on comparing $\beta_{\text {cruise }}$ and $\beta_{\text {plume }}$.

\section{FEEDING RATE OVER A RANGE OF PARTICLE SIZES}

\section{Particle size distributions}

Animals feed on a range of particle sizes whose relative contributions to the animal diet depends on the particle size distributions as well as the relative efficiencies for feeding on the particles. Falling particles occur with a range of sizes which can be represented with particle size distribution $n(r)$ (e.g. Jackson et al. 1997).

Expressed mathematically, the rate at which an animal encounters particles with sizes between $r$ and $r+\mathrm{d} r$ is $\beta n \mathrm{~d} r$, where $\beta$ can be the encounter rate kernel for any search mechanism. If the amount eaten at each contact is $g$, then the total rate of food consumption $F$ is given by:

$$
F=\int_{r_{L}}^{r_{U}} \beta n g \mathrm{~d} r
$$

where $r_{L}$ and $r_{U}$ are the lower and upper size ranges for particle feeding.

There are different strategies that animals use when feeding on the particles. Some animals find an aggre-

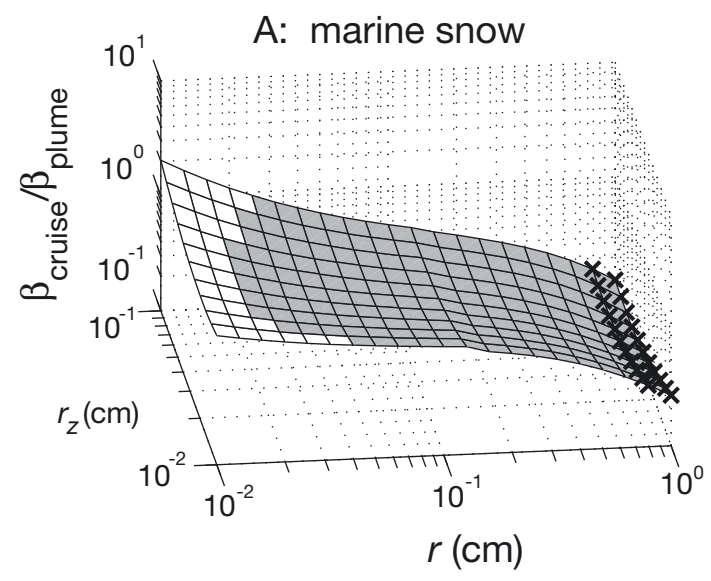

gate, fill their guts, and move off the particle, a strategy called mining; other animals eat the entire particle, engulfing it. Small animals tend to mine. For mining, $g=g_{0}$, where $g_{0}$ is a constant; for engulfing, $g=m(r)$, where $m$ is the mass of the particle. In the case of mining, Eq. (16) simplifies to:

$$
F=\int_{r_{L}}^{r_{U}} \beta n \mathrm{~d} r
$$

while for engulfing:

$$
F=\int_{r_{L}}^{r_{U}} \beta n m \mathrm{~d} r
$$

\section{Numerical results}

Because particle properties and size distributions vary, it is impossible to reach definitive conclusions about the rates at which zooplankton and particles interact. We can, however, choose representative sets of properties. For example, particle abundance, sinking speed, mass, and leakage rate are frequently described by power-law expressions:

$$
\begin{aligned}
& n=a r^{-b} \\
& v=c r^{d} \\
& m=e r^{f} \\
& L=\operatorname{lm}=l e r^{f}
\end{aligned}
$$

where 1 is the specific leakage rate (e.g. Kiørboe \& Jackson 2001). We assume that the animal swims at a rate proportional to its length: $v_{Z}=2.9$ body lengths $\mathrm{s}^{-1}=5.8 r_{Z}$ (body lengths $\left.\mathrm{s}^{-1}\right)(\mathrm{n}=51$; data from Mauchline 1998), $S=r_{Z}$, and that $C_{0}=0.5 \times 10^{-10} \mathrm{~mol} \mathrm{~cm}^{-3}$ (Kiørboe \& Thygesen 2001 and references therein). For the size spectra of both marine snow and fecal pellets,

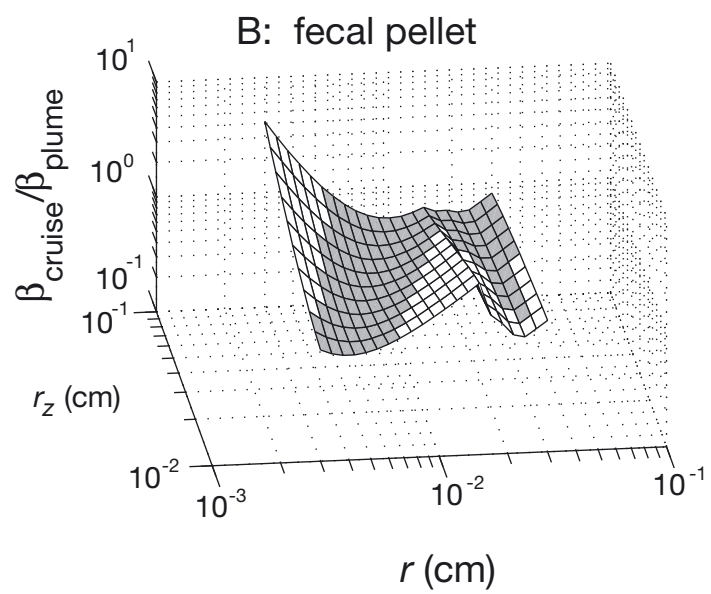

Fig. 5. Comparison of the $\beta$ s for plume seekers relative to cruisers as functions of zooplankton and particle radii. (A) Parameters typical of marine snow particles: $v=0.13 r^{0.26} \mathrm{~cm} \mathrm{~s}^{-1}, L=1 \times 10^{-12} \mathrm{r}^{1.5} \mathrm{~mol} \mathrm{~s}^{-1}$ (Kiørboe \& Jackson 2001); $\mathbf{X}$ : plume length > $1 \mathrm{~m}$. (B) Parameters typical of fecal pellets, with $v$ calculated assuming Stokes sinking with an excess density of $0.125 \mathrm{~g} \mathrm{~cm}^{-3}$

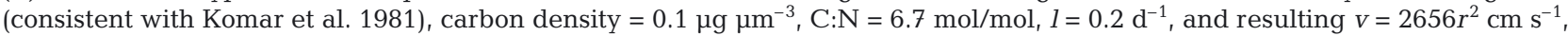
$L=1.21 \times 10^{-8} r^{3} \mathrm{~mol} \mathrm{~s}^{-1}$. In both cases, $C_{0}=0.5 \times 10^{-10} \mathrm{~mol} \mathrm{~cm}{ }^{-3}, u_{*}=0.01 \mathrm{~cm} \mathrm{~s}^{-1}$ (Titelman 2001, Lenz \& Hartline 1999$)$, and $r$ is in $\mathrm{cm}$. Light areas indicate conditions where the $\beta_{\text {cruise }}$ is greater than $\beta_{\text {plumei }}$ dark areas indicate the reverse 

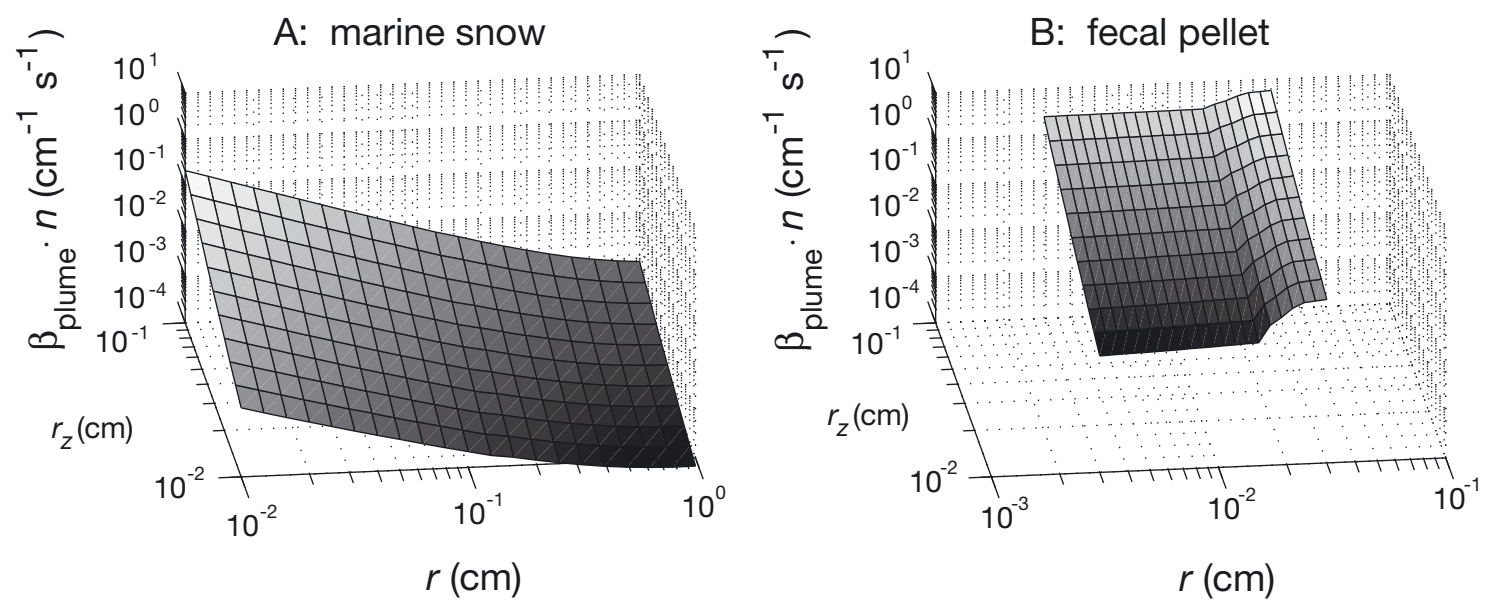

Fig. 6. Number feeding rate spectrum for zooplankton consuming a fixed amount of a particle per visit $\left(\beta_{\text {plume }} n\right)$. This can be converted into the mass feeding rate spectrum similar to that shown in Fig. 7 by multiplying with the mass eaten per visit $\left(g_{0}\right)$.

(A) Particle distribution and types typical of marine snow; (B) particle typical of fecal pellets. Values as in Fig. 5

we use values observed in Monterey Bay, California, $\left(a=2.5 \times 10^{-5}\right.$ and $b=3$; Jackson et al. 1997). These certainly greatly overstate the concentration of fecal pellets but provide a starting point for discussion.

For typical particle characteristics of marine snow and fecal pellets, the comparison shows that plume-finding is more effective than cruising but that the differences between the 2 strategies are relatively slight (Fig. 5). While there is a small effect of animal size, there is a much greater effect of particle size and settling rate. For a particle characteristic of marine snow, the relative efficiency of plume-finding exceeds that of cruising for all but the smallest particles, in part because of the ever lengthening plume (Fig. 5A). For the largest particles, the plume can exceed $1 \mathrm{~m}$. It is unknown how long such a plume can become before other processes such as turbulence disrupt it. A small particle characteristic of a fecal pellet is also more efficiently found by the cruiser, but larger particles are more efficiently found by the plume finders (Fig. 5B). More interesting than the differences between different-sized fecal pellets is the fact that the 2 particle finding modes are so similar.

The feeding rate spectrum for plume finding $\left(=\beta_{\text {plume }} n\right)$ suggests that it is easier to find a small marine snow-type particle than a large one, but that fecal pellet-type particles are easiest to find and that large and small pellets are encountered at similar rates (Fig. 6). The relative spectral shape shifts to favoring larger particles if the greater mass of the larger particles is included ( $\beta_{\text {plume }} n m_{i}$ Fig. 7 ). For the marine snow particles, the mass feeding rate spectrum is essentially flat, whereas for fecal pellets the larger ones are favored. The greater swimming rates and sensory ranges favor the larger animals.

The feeding rate spectra describe the relative importance of each size class in the potential diet of an ani-
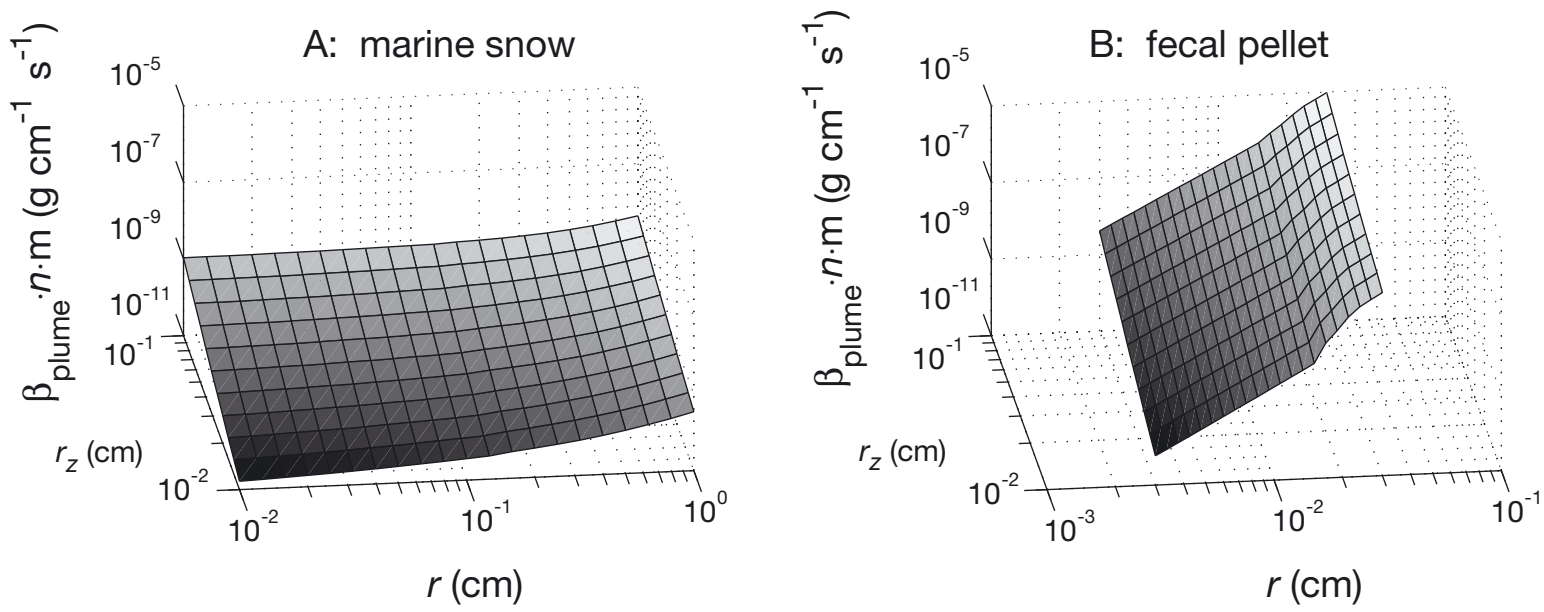

Fig. 7. Mass feeding rate spectrum for zooplankton consuming the entire particle. (A) Particle distribution and types typical of marine snow; (B) particle typical of fecal pellets. Values as in Fig. 5 

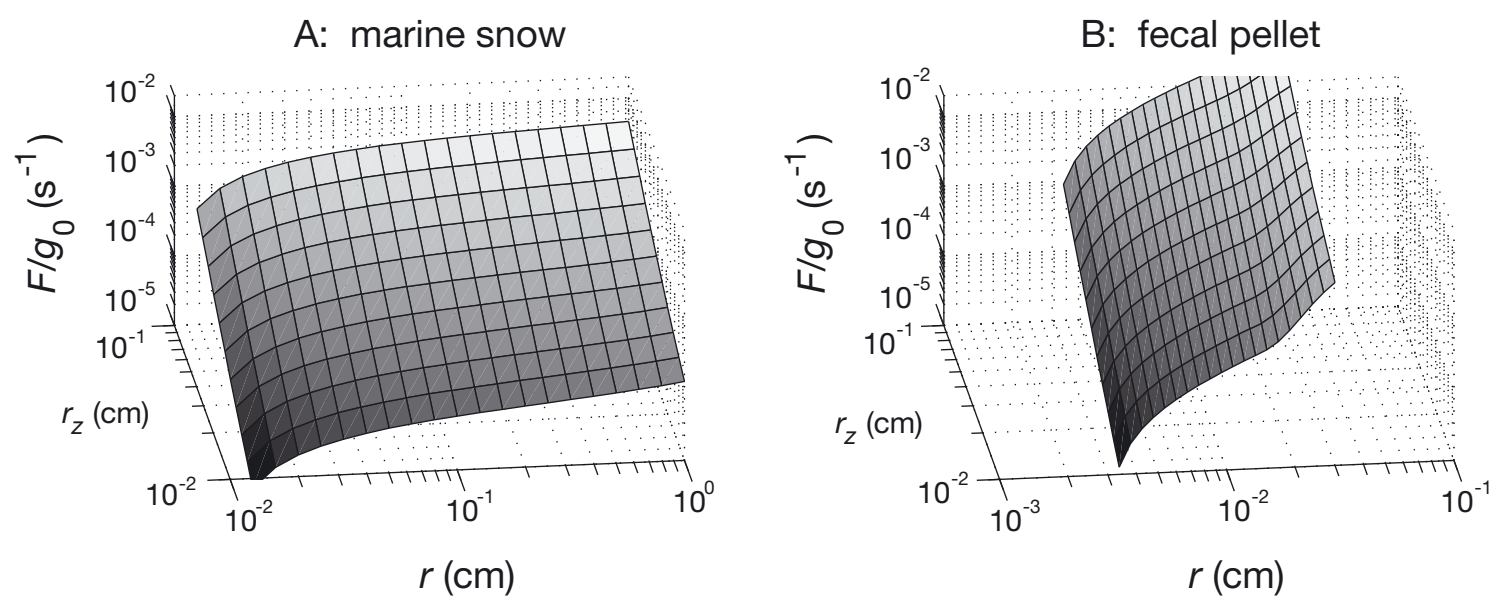

Fig. 8. Integrated feeding rate divided by the food ration for a zooplankter consuming a fixed amount per visit to the particle $\left(\int_{r_{L}}^{T} \beta_{\text {plume }} n d r=F / g_{0}\right)$. When multiplied by the food ration, it represents the total rate of food concentration when eating from particles between $r_{L}=30 \mu \mathrm{m}$ and $r$ in size. (A) Particle distribution and types typical of marine snow; (B) particle typical of fecal pellets. Values as in Fig. 6 . The maximum amount that an animal can eat is $3 \times$ the fecal pellet volume. The relationship between fecal pellet volume and prosome length is $\log V_{F}=2.474 \log L_{P}+5.226$ where $V_{F}$ is fecal pellet volume $\left(\mu m^{3}\right)$ and $L_{P}$ is prosome length (mm), using data for copepods from Mauchline (1998). We estimate the prosome as $3 \times$ the zooplankton radius

mal. Integrating the feeding rate spectra provides a measure of the total food available for an animal (Figs. 8 \& 9). The quantity $F / g_{0}$ provides a contact rate for the mining zooplankter, with the inverse providing an estimate for the time between contacts. For the larger animals, the contact time between aggregates could be less than $220 \mathrm{~s}$ for the marine snow particles and $10 \mathrm{~s}$ for the fecal pellets (Fig. 8).

The integrated feeding rate for the animals that consume the entire particle is heavily weighted toward the larger particles (Fig. 9). For the largest animals, the consumption levels can be as high as $63 \mathrm{ngC} \mathrm{s}^{-1}$ for fecal pellets and $2.4 \mathrm{ngC} \mathrm{s}^{-1}$ for marine snow, corresponding to, respectively, more than 10 times and

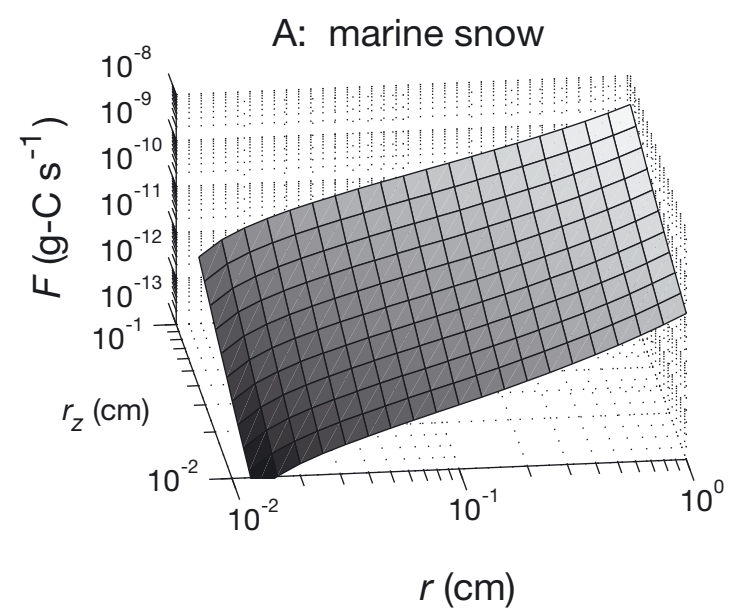

approximately $50 \%$ body carbon $\mathrm{d}^{-1}$. This is a very reasonable number for the marine snow, but too high for the fecal pellets, probably reflecting the greatly overstated fecal pellet concentrations assumed in the calculations.

\section{DISCUSSION}

We emphasized 2 sets of particle properties and distributions as a way of exploring a range of possibilities. However, marine snow particles have a range of mass, fall velocities, and size distributions. These differences will affect the actual rates of zooplankton feeding. The

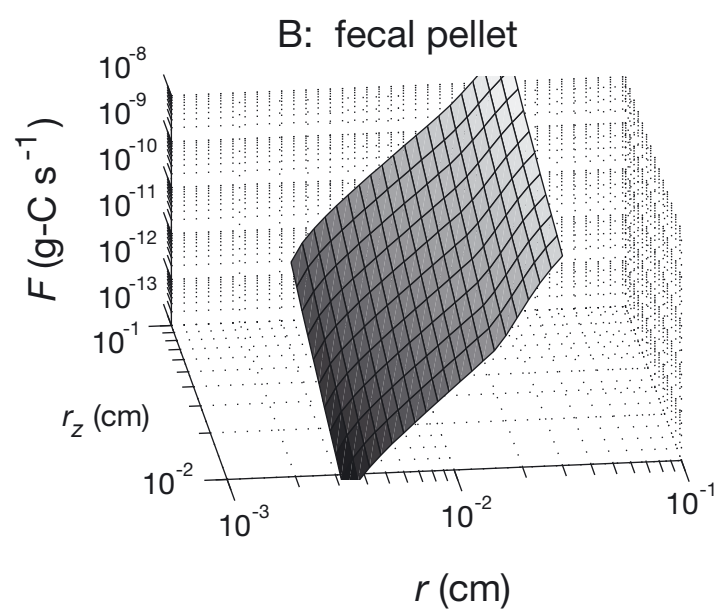

Fig. 9. Feeding rate for a zooplankter consuming the entire particle upon contact. This represents the rate if it consumes particles between $r_{L}=30 \mu \mathrm{m}$ and $r$. (A) Particle distribution and types typical of marine snow; (B) particle typical of fecal pellets. Values as in Figs. $5 \& 7$ 

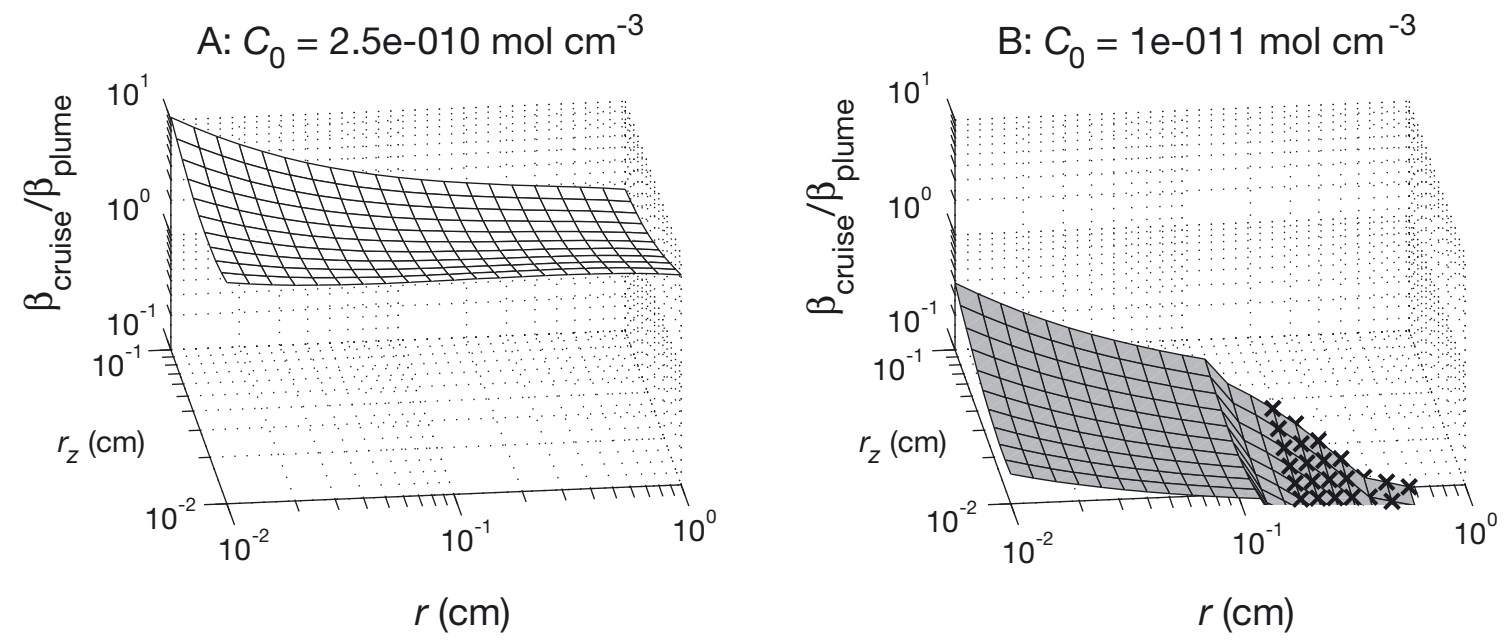

Fig. 10. Effect of threshold concentration on the comparison of the $\beta \mathrm{s}$ for plume seekers relative to cruisers as functions of zooplankton and particle radii. Parameters are the same as those for Fig. 5A, with the exception of the threshold concentrations. (A) $C_{0}=2.5 \times 10^{-10} \mathrm{~mol} \mathrm{~cm}^{-3}$; (B) $C_{0}=1 \times 10^{-11} \mathrm{~mol} \mathrm{~cm}^{-3}$. These are $5 \times$ and $1 / 5$ of the $C_{0}$ used elsewhere

focus of this paper has been on demonstrating that plume feeding can be described relatively simply and that it appears to be a very efficient way for animals to find falling food.

This analysis has focussed only on the effect of feeding strategy on feeding rate, showing the importance of animal size in determining feeding rate. The analysis did not include the costs of the different strategies. These include the metabolic costs of being large and of moving to find particles. The costs also do not include any enhanced mortality rate associated with swimming, which increases the detectability of an animal, as well as increasing the rate at which it collides with an ambush predator. These costs are similar for cruisers and plume finders, but different for sedentary ambush and flux feeders. The final analysis of the value of a feeding strategy for any animal must include these costs as well as the gains.

\section{Comparison of predictions with observations}

While the prediction is that plume finding should be a common strategy, we know very little about the potential for zooplankton to employ this strategy. It has been demonstrated qualitatively for only 2 species, a planktonic shrimp (Hamner \& Hamner 1977) and a planktonic copepod (Kiørboe 2001), and it is known that several copepods can follow pheromone trails to find mates (Tsuda \& Miller 1998, Yen et al. 1998). Such observations are difficult to make. Many more observations have demonstrated that zooplankton can use hydromechanical cues (Haury et al. 1980, Fields \& Yen 1997, Kiørboe et al. 1999). This does not necessarily reflect the relative occurrence of the different strategies.

\section{Plume size}

Experimental observations allow the estimation of the parameters describing particle detection by hydromechanical mechanisms. There are few corresponding observations for plume finding in animals, although there are more such measurements of particle detection by microorganisms (e.g. Blackburn et al. 1998, Mitchell et al. 1996). For example, the value of $C_{0}$ used here could be high. A reasonable first estimate would be that the minimum increment in concentration of an amino acid that is detectable equals its background concentration. A typical value for the sum of all dissolved free amino acids in the ocean is $25 \mathrm{nM}=2.5 \times$ $10^{-11} \mathrm{~mol} \mathrm{~cm}^{-3}$ (e.g. Lee \& Bada 1977). Because the concentration of any single amino acid is less, a value of $C_{0}=10^{-11} \mathrm{~mol} \mathrm{~cm}^{-3}$ is reasonable and one-fifth of the value used in the above simulations. Using this smaller value of $C_{0}$ increases the plume length by a factor of 5 and the $\sigma_{0}$ by $5^{1.5}=11$ (Eqs. $3 \& 5$ ). Higher sensitivities to specific amino acids have been observed in copepods $\left(10^{-12} \mathrm{~mol} \mathrm{~cm}^{-3}\right.$; Yen et al. 1998) and even greater sensitivities have been reported for marine crustaceans (e.g. Fuzessery \& Childress 1975, Olla 1977, Pearson et al. 1980). The resulting increases in contact rates for plume feeders further enhance the advantage of this mode of particle feeding. Conversely, an increase in the threshold concentration would decrease the contact rates.

As an example, the results for the conditions used here suggest that plume-sensing and cruising are generally similar in the rates of zooplankton contact with marine snow, although cruising is more effective for smaller particles and plume sensing more effective for larger ones (Fig. 5). Increasing the threshold concen- 
tration by a factor of 5 makes the cruising mode more effective (Fig. 10A); decreasing the threshold concentration by a factor of 5 makes plume-sensing more effective (Fig. 10B). There is enough variability in the properties and distributions of natural particles to make a simple generalization impossible, but the comparison is interesting.

The other important factor that we have simplified is the rate of particle solubilization. It can be expected to vary with the state of decay of the particle, as well as anything that changes bacterial metabolic rates, such as temperature.

Turbulence is an important process that has not been included in this analysis. It could be expected to decrease the plume length by disrupting the integrity of the plume, thereby decreasing the rate of particle feeding. The intermittent nature of turbulence affects both the time interval that a plume exists as well as its total length. As a result, a rapidly falling particle should have its plume less disrupted by turbulence than an equal length plume from a slowly settling particle.

\section{Particle distributions and properties}

The properties of the sinking particles are crucial to the encounter rates. While there are numerous observations on particle size distributions as a function of diameter, there are substantially fewer on the simultaneous variations in particle mass. Simultaneous in situ measurements of particle settling speed and diameter have shown considerable variability in the average properties at different locations as well as in the properties at any one time and place. Examples where this has been done include the settling measurements of Alldredge \& Gotschalk (1988) and Asper (1987) as well as settling spectra determined from sediment traps (Kiørboe et al. 1996, Waite \& Nodder 2001). Previous theoretical studies of aggregate generation suggest that the multiple particle sources in the environment can affect the mass and settling properties of particles, so that they need to be characterized by multiple measurements on the same particles (Jackson 1998, 2001). Because particle settling speed depends on both mass and diameter, the implication is that there is considerable variation in particle composition.

Our use of a hypothetical distribution for fecal pellets in this analysis was an attempt to broaden the expected range of particle behaviors. It shows that particle type can be important in determining the relative success of different feeding behaviors. In particular, hydrodynamic sensing should be most efficient at sensing the most massive particles, such as large fecal pellets (Svensen \& Kiørboe 2000). In contrast, plume finders should be more effective with the other particles.

The importance of both particle mass and diameter for animal feeding suggests that they are important properties to measure. Among the possible approaches are the determination of the rate of fecal pellet production and the pellet properties (e.g. Feinberg \& Dam 1998).

\section{Effect of depth}

Depth provides a dramatic range of environments over relatively short distances for planktonic animals. This range has implications for different particle feeding types. Aside from the decrease in particle flux and concentration with depth, there are changes in the physical environment and in the nature of the particles.

Turbulence levels decrease with depth (e.g. Gargett \& Osborn 1981). As a result, there should be less disruption of the plume structure, allowing longer plumes and more effective plume finding. Less turbulence also implies less hydrodynamic noise and, hence, more efficient detection of hydrodynamic signals.

In addition, the rate of aggregation should decrease with depth as a result of the lower particle concentrations and lower turbulent shear conditions. This should change the slope of the particle size distributions. The relative contribution of fecal pellets to the fluxing particulate material also decreases with depth, since fecal pellets are remineralized rapidly in the upper water column (e.g. Smetacek 1980, Riser et al. 2001).

Because the background concentrations of organic matter decrease with depth (e.g. Lee \& Bada 1977), there may be a decrease in the concentration enhancement needed for plume detection. These changes would lead to an increase with depth of the relative advantage of the plume-finding strategy over the cruising strategy. This might be partially compensated by a change with depth of the composition of the falling particles, which includes a decrease in the relative concentration of labile organic matter (e.g. Armstrong et al. 2001) and a consequent weakening of the resulting chemical signal.

Lastly, the decrease in zooplankton abundance with depth should reduce the predation pressure and increase the value of active vs passive strategies.

The conclusion from these considerations is that the efficiency of particle feeding should have a strong depth component. Overall, the prediction is that the relative advantage of plume finders is expected to increase with depth, while cruisers are at a relative advantage near the surface. 
Acknowledgements. This work was supported by grants OCE9986765 and OCE9981424 from US-JGOFS program in the Ocean Sciences Division of NSF to G.A.J. and the Danish Natural Science Research Council, contract \# 21-01-0549 to T.K. We are grateful to A. Visser and U. Thygesen for helpful discussions.

\section{LITERATURE CITED}

Alldredge AL, Gotschalk C (1988) In situ settling behavior of marine snow. Limnol Oceanogr 33:339-351

Armstrong RA, Lee C, Hedges JL, Honjo S, Wakeham SG (2001) A new, mechanistic model for organic carbon fluxes in the ocean, based on the quantitative association of POC with ballast minerals. Deep-Sea Res II 219-236

Asper VL (1987) Measuring the flux and sinking speed or marine snow aggregates. Deep-Sea Res 34:1-17

Blackburn N, Fenchel T, Mitchell J (1998) Microscale nutrient patches in planktonic habitats shown by chemotactic bacteria. Science 282:2254-2256

Feinberg LR, Dam HG (1998) Effect of diet on dimensions, density, and sinking rates of fecal pellets of the copepod Acartia tonsa. Mar Ecol Prog Ser 175:87-96

Fields DM, Yen J (1997) The escape behaviour of marine copepods in response to a quantifiable fluid mechanical disturbance. J Plankton Res 19:1298-1304

Fischer HB, Imberger J, List EJ, Koh RCY, Brooks NH (1979) Mixing in inland and coastal waters. Academic Press, New York

Fuzessery ZM, Childress JJ (1975) Comparative chemosensitivity to amino acids and their role in the feeding activity of bathypelagic and littoral crustaceans. Biol Bull 149: 522-538

Gargett AE, Osborn TR (1981) Small-scale measurement during the Fine and Microstructure Experiment (FAME). J Geophys Res 86(C3):1929-1944

Hamner P, Hamner WM (1977) Chemosensory tracking of scent trails by the planktonic shrimp Acetes sibugae australis. Science 195:886-888

Haury LR, Kenyon DE, Brooks (1980) Experimental evaluation of the avoidance reaction of Calanus finmarchicus. J Plankton Res 2:187-202

Jackson GA (1989) Simulation of bacterial attraction and adhesion to falling particles in an aquatic environment. Limnol Oceanogr 34:514-530

Jackson GA (1993) Flux feeding as a mechanism for zooplankton grazing and its implications for vertical particulate flux. Limnol Oceanogr 38:1328-1331

Jackson GA (1998) Using fractal scaling and two dimensional particle size spectra to calculate coagulation rates for heterogeneous systems. J Colloid Interface Sci 202:20-29

Jackson GA (2001) Effect of coagulation on a model planktonic food web. Deep-Sea Res I 48:95-123

Jackson GA, Maffione R, Costello DK, Alldredge AL, Logan BE, Dam HG (1997) Particle size spectra between $1 \mu \mathrm{m}$ and $1 \mathrm{~cm}$ at Monterey Bay determined using multiple instruments. Deep-Sea Res I 44:1739-1767

Kiørboe T (2001) Formation and fate of marine snow: smallscale processes with large-scale implications. Sci Mar 65(Suppl 2):57-71

Kiørboe T, Jackson GA (2001) Marine snow, organic solute

Editorial responsibility: Otto Kinne (Editor),

Oldendorf/Luhe, Germany plumes, and optimal chemosensory behavior of bacteria. Limnol Oceanogr 46:1309-1318

Kiørboe T, Thygesen UH (2001) Fluid motion and solute distribution around sinking aggregates. II. Implications for remote detection by colonizing zooplankters. Mar Ecol Prog Ser 211:15-25

Kiørboe T, Visser AW (1999) Predator and prey perception in copepods due to hydromechanical signals. Mar Ecol Prog Ser 179:81-95

Kiørboe T, Hansen JLS, Alldredge AL, Jackson GA and 5 others (1996) Sedimentation of phytoplankton during a spring diatom bloom: rates and mechanisms. J Mar Res 54:1123-1148

Kiørboe T, Saiz E, Visser A (1999) Hydrodynamic signal perception in the copepod Acartia tonsa. Mar Ecol Prog Ser 179:97-111

Komar PD, Morse AP, Small LF, Fowler SW (1981) An analysis of sinking rates of natural copepod and euphausiid fecal pellets. Limnol Oceanogr 26:172-180

Lee C, Bada JL (1977) Dissolved amino acids in the equatorial Pacific, the Sargasso Sea, and Biscayne Bay. Limnol Oceanogr 22:502-510

Lenz PH, Hartline DK (1999) Reaction times and force production during escape behavior of a calanoid copepod, Undinula vulgaris. Mar Biol 133:249-258

Mauchline J (1998) The biology of calanoid copepods. Academic Press, San Diego

Mitchell JG, Pearson L, Dillon S (1996) Clustering of marine bacteria in seawater enrichments. Appl Environ Microbiol 62:3716-3721

Okubo A (1980) Diffusion and ecological problems: mathematical models. Springer-Verlag, Berlin

Olla BL (1977) Chemoreception in the blue crab, Callinectes sapidus. Biol Bull 153:346-354

Pearson WH, Thomson H, Ache BW (1980) Threshold determination of olfactory receptors of the spiny lobster. Mar Behav Physiol 7:249-260

Riser CW, Wassmann P, Olli K, Arashkevich E (2001) Production, retention and export of faecal pellets on and off the Iberian shelf, north-west Spain. Prog Oceanogr 51:423-441

Smetacek V (1980) Annual cycle of sedimentation in relation to plankton ecology in western Kiel Bight. Ophelia (Suppl) 1:65-76

Svensen C, Kiørboe T (2000) Remote prey detection in Oithona similis: hydromechanical versus chemical cues. J Plankton Res 22:1155-1156

Thygesen UH, Kiørboe T (2002) A Matlab environment for analysis of fluid flow and transport around a translating sphere. Mar Models 2:35-36

Titelman J (2001) Swimming and escape behavior of copepod nauplii: implications for predator-prey interactions among copepods. Mar Ecol Prog Ser 213:203-213

Tsuda A, CB Miller (1998) Mate-finding behavior in Calanus marshallae Frost. Phil Trans R Soc Lond B 353:713-720

Visser AW (2001) Hydromechanical signals in the plankton. Mar Ecol Prog Ser 222:1-24

Waite AM, Nodder SD (2001) The effect of in situ iron addition on the sinking rates and export flux of Southern Ocean diatoms. Deep-Sea Res II 48:2635-2654

Yen J, Weissburg MJ, Duall MH (1998) The fluid physics of signal perception by mate-tracking copepods. Phil Trans R Soc Lond B 353:787-804

Submitted: November 19, 2002; Accepted: October 11, 2003

Proofs received from author(s): March 4, 2004 